\title{
LA RELACIÓN A FINALES DEL PERÍODO HORIZONTE TEMPRANO E INTERMEDIO TEMPRANO EN CONVENTOMOQO, VALLE DEL CUSCO
}

\author{
Carlos Delgado González \\ Universidad NaCIONAL de SAN ANTONIO ABAD del CUSCO \\ mauriciodel@Hotmail.com
}

\section{RESUMEN}

Este trabajo trata sobre la interacción de los estilos cerámicos Chanapata Derivado y los estilos del Período Intermedio Temprano como Qotakalli y una cerámica "Local" durante este período e inicios del Horizonte Medio en el sitio arqueologico de Conventomoqo en el valle del Huatanay.

Conventomoqo es un lugar interesante donde se tiene una columna estratigráfica desde el Período Horizonte Temprano hasta la época Inca, lugar que inicialmente fue identificado por Bauer en su prospección intensiva del valle del Cusco (2008) y por el Instituto Nacional de Cultura del Cusco. Las excavaciones arqueológicas nos han permitido obtener algunos derroteros para plantear que el estilo ceramico Chanapata Derivado siguio produciendose y utilizandose en Conventomoqo cuando el estilo Qotakalli se encontraba densamente difundido en el valle del Huatanay. Ademas los datos de la excavación y el análisis de la cerámica nos ha conducido a plantearnos una interrogante sobre un estilo de cerámica a la que denominamos "Local” para el valle del Cusco y sería contemporánea con Qotakalli.

Palabras claves: Chanapata Derivado, Qotakalli, Período Intermedio Temprano.

\section{AbSTRACT}

This work treats about the interaction between the ceramic styles Derived Chanapata and the styles of the Early Intermediate Period like Qotakalli and a "local" ceramic during this period and the beginning of the Middle Horizon in the archaeological site Conventomoqo in the Huatanay valley.

Conventomoqo is an interesting site where a stratigraphic sequence from the Early Horizon to the Inca period exists. The site was first identified by Bauer in his extensive prospection of the Cusco valley (2008) and by the Nacional Institute of Culture of Cusco. The archaeological excavations allowed us to obtain indications to propose that the Derived Chanapata style was still being produced 
and used in Conventomoqo when the Qotakalli style was densely diffused in the Huatanay valley. Furthermore the results of the excavation and the analysis of the ceramic led us to put a question mark on a ceramic style that we call "local" for the valley of Cusco and if it could be contemporary with Qotakalli.

KeYwords: Derived Chanapata, Qotakalli, Early Intermediate Period.

\section{INTRODUCCIÓN}

El valle del Cusco ha sido densamente ocupado desde los 5000 años a.C, con los primeros habitantes durante el período Arcaico y tiene una ocupación continuada hasta hoy en día. Los primeros grupos sedentarios en el valle fueron los que utilizaban el estilo de cerámica que se ha denominado Marcavalle y posteriormente el estilo Chanapata que ocuparon ampliamente todo el valle con poblaciones medianas y pequeñas que han sido identificadas desde la década del 1940 (Barreda 1973, 1982, Dwyer 1971, Chávez 1980, 1981a, 1981b, 1982, Rowe 1944, Yabar 1959, 1972, 1982, y Zapata 1998). Seguidamente hubo la ocupación Qotakalli que ha sido identificada en el valle del Cusco, Anta y el Valle Sagrado con evidencias de sitios en las partes bajas de los valles (Barreda 1973, Kendall 1976, Espinoza 1983, McEwan 1984, Torres 1989, Glowacki 1996, Zapata 1997, Bauer 2002, 2008, Bauer y Jones 2011, Covey 2003, Covey et. al. 2008, Bélisle y Galiano 2010, Bélisle y Covey 2010 y Bélisle 2011). El cambio entre estos dos periodos todavia los arqueólogos no hemos podido esclarecer con claridad la transición entre ambos períodos y tenemos algunos vacios para definir aspectos temporales, espaciales y de estilo.

\section{Conventomoqo y el VAlle del Cusco}

Sitio arqueológico que se ubica en la ladera del cerro Contaymoqo a la margen derecha del río Huatanay, en el distrito de San Jerónimo, departamento y provincia del Cusco a $3250 \mathrm{~m}$ de altitud, entre las coordenadas UTM 186070 Este y 8501200 norte. Actualmente el lugar se encuentra ocupado integramente por la Asociación de Vivienda Altiva Canas.

El valle del Huatanay corresponde a un área de Bosque Seco Montano Subtropical, como elemento principal se tiene al río Huatanay. Gran parte del valle del Cusco fue una zona inundable y no fue adecuado para una ocupacción humana permanente (Bauer 2008:21). Las zonas agricolas más productivas son las grandes terrazas aluviales de la parte sur que se encuentran sobre los $25 \mathrm{~m}$ del piso de valle y son aptas para el cultivo de diferentes productos como el maíz que se cultiva hasta los 3600-3800 m, la papa se cultiva hasta los $4300 \mathrm{~m}$., la quinua entre los 2000 a 3600 que se siembra generalmente con el maíz (Ugent y Ochoa 2006:104). Esta práctica de cultivar maíz y quinua conjuntamente se puede ver entre San sebastián y San Jerónimo, en los andenes Larapa y al sur de Conventomoqo. Asimismo, se continua sembrando la oca en las laderas del valle del Huatanay, cultivo que se siembra hasta los $4000 \mathrm{~m}$ de altitud.

\section{LAS eXCAVACiones aRqueológicas en ConVentomoqo}

Conventomoqo es una colina de 4 hectáreas que fue modificada parcialmente haciendola relativamente plana de $300 \mathrm{~m}$ de largo por $140 \mathrm{~m}$ de ancho, esta es una característica que ya se tiene documentada para otros sitios del período Formativo (ver Bauer 2008:88, Zapata 1998:320, Davis y Delgado 2010:356). 
El año 1997 realizamos un trabajo de arqueología de salvataje en el sitio arqueológico que se encontraba en proceso de ocupación por la Asociación de Vivienda Altiva Canas (ver figura 1). El área donde pudimos trabajar correspondió a las áréas libres como parques, calles y algunos lotes de vivienda que todavía no habían sido construidos. Las excavaciones arqueológicas demostraron que el lugar tuvo una ocupación continuada desde el período Formativo Tardío (asociado a cerámica Chanapata derivado) hasta el período Inca, con una fuerte presencia de cerámica Qotakalli.

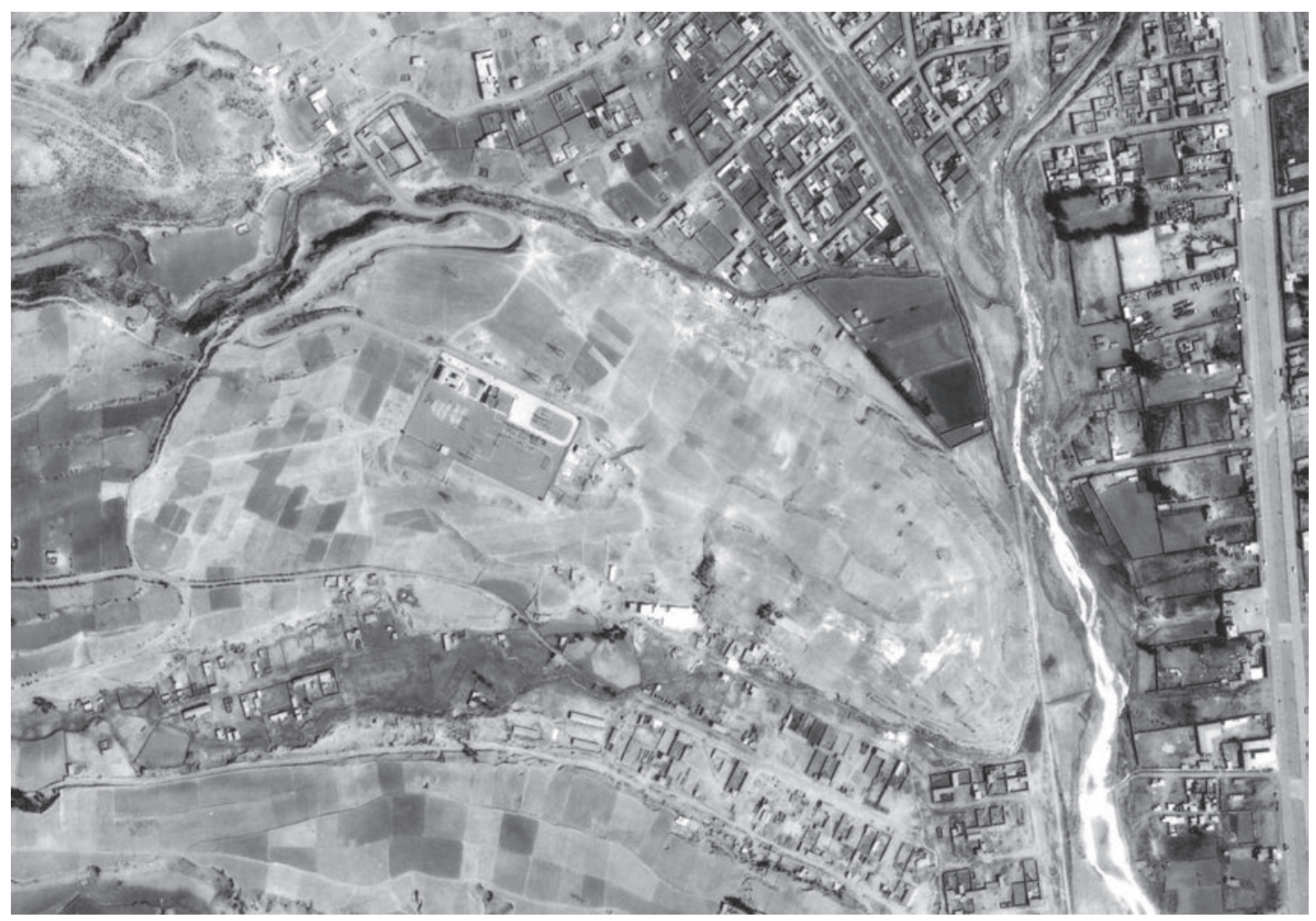

Figura 1: Foto aérea del Instituto Geográfico Nacional del sitio de Conventomoqo en 1997.

\section{LA OCUPACIÓN DURANTE EL HORIZONTE TEMPRANO EN CONVENTOMOQO}

Es durante el período Horizonte Temprano que el valle del Huatanay tiene un fuerte crecimiento con varios asentamientos grandes que se establecen a la margen derecha e izquierda del río Huatanay. La primera gran ocupación fue en el sitio de Marcavalle que fue descubierto en 1953 por Manuel Chávez Ballón y Jorge Yábar Moreno y luego excavado (Barreda 1973:24, Chávez 1980, 1982). Karen Mohr Chávez logró determinar una ocupación desde 1000 a 700 años a.C (Chávez 1980:240, 1982:2).

Posteriormente a Marcavalle tenemos una ocupación asociada a cerámica Chanapata y Chanapata Derivado (700 años a.C a los 100 años d.C), que fue identificada primeramente por John Rowe (1944) en la colina de Carmenca (actual barrio de Santa Ana), este estilo es el que se encuentra prolíficamente tanto en el valle del Huatanay, valle Sagrado y la Pampa de Anta (Bélisle y Galiano 2010, Bélisle y Covey 2010 y Bélisle 2011, Delgado 2014). 
Durante las excavaciones arqueológicas en Conventomoqo se llegó a determinar varios eventos asociados a cerámica Chanapata y Chanapata Derivado. La primera evidencia la encontramos en la unidad de excavación № 13 llegando a encontrar un fogón y varias intrusiones irregulares sobre el suelo geológico, asociadas a un entierro secundario de un neonato desarticulado, incompleto de sexo indeterminado ${ }^{1}$, para lo cual se excavó una cavidad sobre el suelo geológico de $0.48 \mathrm{~m}$ de diámetro y una profundidad de $0.25 \mathrm{~m}$, no estuvo asociado a ninguna ofrenda.

En las unidades de excavación № 15 y 17 se tiene la mayor cantidad de eventos de este período, asociados a fogones y áreas de combustión asociadas a ceniza, puntos de carbón y fragmentería de cerámica Chanapata Derivado. Asimismo, el área presenta varias intrusiones sobre el suelo geológico, una de estas es la intrusión 3 (ver Figura $\mathrm{N}^{\circ}$ 2), que tiene un área de 1.30 por $1.0 \mathrm{~m}$, donde el análisis botánico determinó 24 granos de quinua, 97 de kiwicha, y granos enteros de maíz ${ }^{2}$ También esta intrusión contiene una estructura doméstica que es muy similar a las intrusiones E y H encontradas en Yuthu (Davis y Delgado 2010: fig.10:359) y a la encontrada en Bandojan (Delgado 2014).

En los diferentes estratos del Horizonte Temprano se identificaron varias especies de origen silvestre en la muestra 2 (se identificó fragmentos de semilla de la familia Fabaceae, un fruto carbonizado de Asteraceae así como 14 fragmentos de semilla de la familia Solanaceae ${ }^{3}$ ), en la muestra 3 (7 frutos carbonizados de origen silvestre de Asteraceae Asteraceae y 22 semillas, además de 24 fragmentos de semilla de la familia solanaceae $e^{4}$ que podrían corresponder a semillas de papa o ají silvestre.

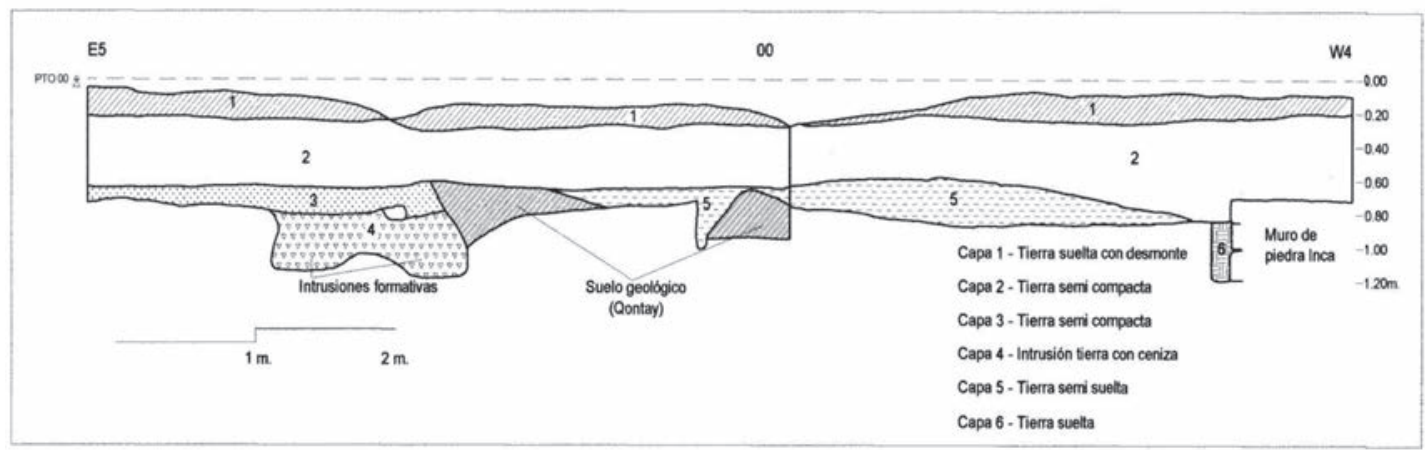

Figura 2: Perfil estratigráfico sur de la Unidad de excavación 17

El análisis cerámico de la Unidades 15 y 17 determino que la cerámica Chanapata llana y Chanapata bruñido por patrones tiene $94.5 \%$, Chanapata Blanco /rojo 3.75\%, 1.44\% Incisa y $0.31 \%$ las aplicaciones plásticas. La morfología de la cerámica corresponde generalmente a formas restringidas como las ollas y jarras y en menor proporción a platos y cuencos.

En la unidad de excavación № 20 se construyeron algunos alineamientos de piedra y algunas intrusiones sobre el suelo geológico con un relleno de tierra con carbón asociado a fragmentería de cerámica Chanapata Derivado. El análisis botánico determin\{o 2 semillas y 1 fragmento de semilla carbonizados de la familia Solanaceae; 14 fragmentos de tallos carbonizados de la familia Magnolióp-

1. Análisis realizado por Ph. D Valerie Andrushko.

2. Rojas Velásquez 2011:Muestra № 01. 
sida $a^{5}$ asociados al entierro $\mathrm{N}^{\circ} 2$, lo cual tenía un marcador de una piedra parada encima del entierro, la matriz del entierro corresponde a una intrusión circular sobre el suelo geológico de $0.50 \mathrm{~m}$ de diámetro a una profundidad de $0.60 \mathrm{~m}$. El individuo de sexo femenino de aproximadamente 35 años de edad colocado en posición flexionada con la cabeza orientada al norte tenía asociado 4 piedras a su alrededor (ver figura 3), el individuo presenta 8 piezas dentales con caries, 8 dientes se perdieron en vida (los alveolos se encuentran cerrados); el cráneo tiene trauma (fractura) y presenta periostitis ${ }^{6}$, asimismo presenta evidencias de osteoartritis en las articulaciones vertebrales.

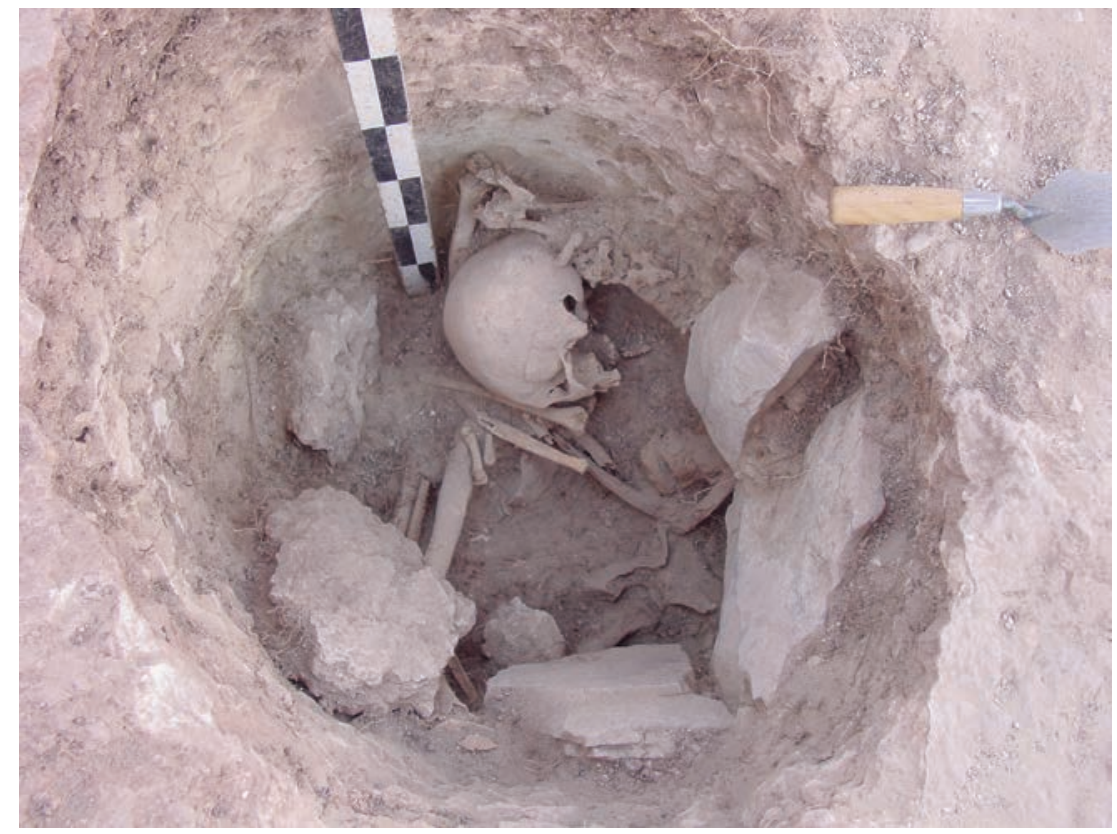

Figura 3: Cista con un entierro de un adulto en posición flexionada.

El análisis del material cerámico en Conventomoqo determinó que en esta área no existián sectores diferenciables, el material cerámico de las diferentes unidades de excavación es similar, la proporción de cerámica decorada es mínima con relación al material doméstico. La cerámica doméstica o utilitaria es llana generalmente de formas restringuidas como ollas, jarras y cantaros. La cerámica Chanapata blanco sobre rojo generalmente se encuentra en platos grandes, cuencos y vasos (ver figura 6).

En los análisis del material cerámico en los sitios con cerámica Chanapata es consistente la presencia de ollas sin cuello (Davis y Delgado 2010, Chávez 1980, 1981a, 1981b, Belisle 2011) y en Bandojan (Delgado 2013). Es sumamente interesante notar que existe una escasez de este tipo de ollas en Conventomoqo, podríamos suponer que en Conventomoqo este tipo es más recurrente en el período formativo y va disminuyendo su producción y uso hacia el período Intermedio Temprano, lo que se debe indicar que en otros sitios como Ak'awillay no se nota estos cambios y su producción es similar.

5. Bertoni 2012: Muestra 5

6. Periostitis, proceso de inflamación del hueso periostio en las tibias (análisis realizado por Ph.D Valerie Andrushko). 


\section{La Ocupación del Período Intermedio Temprano en Conventomoqo}

En Conventomoqo se llegó a identificar varios contextos con cerámica Qotakalli asociados a cerámica Chanapata Derivado. Es así que en la unidad de excavación 3 (ver figura 5), el material cerámico recuperado ${ }^{7}$ nos proporcionó información que más del $50 \%$ corresponde a cerámica doméstica local, los estilos Chanapata Derivado y Qotakalli bordean el 20\% cada uno y se encuentran uniformemente en todas las capas. El estilo denominado "Local" por sus semejanzas con la cerámica local que determino Belisle y Galiano (2010) en la Pampa de Anta, se encuentra en los estratos 4, 5 y 6. El estilo Arahuay se encuentra en una proporción de 1,5\% en todos los niveles, a diferencia que los estilos Muyu Orqo y Horizonte Medio que se encuentran en los estratos más tardíos (ver figura 4).

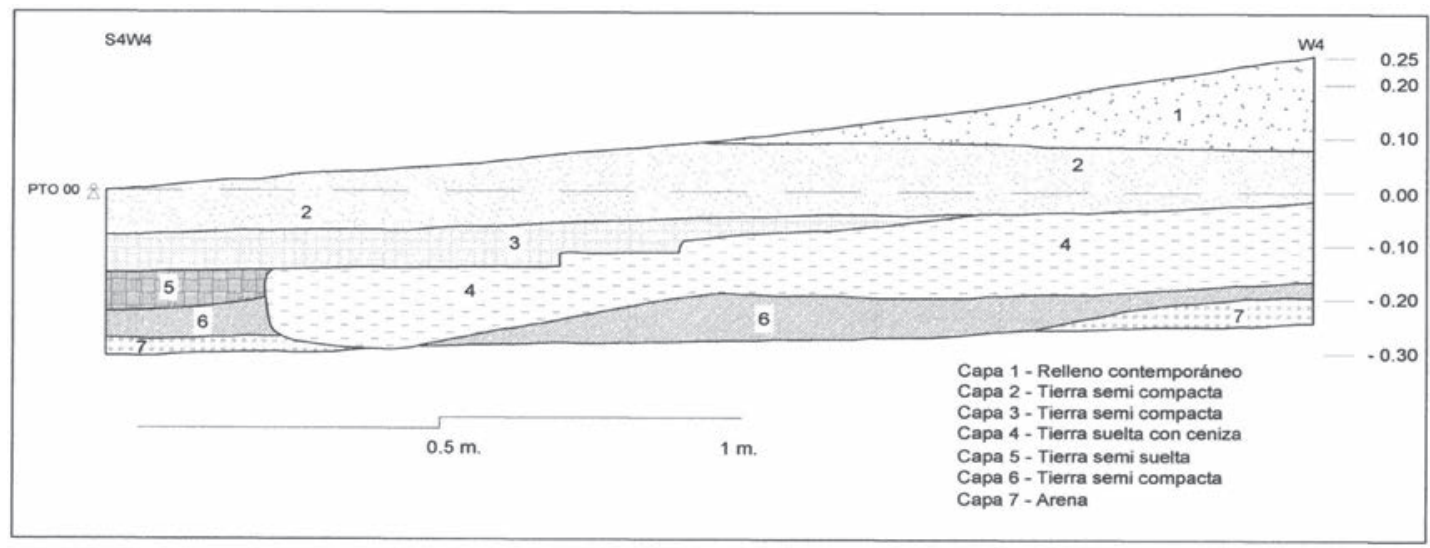

Figura 4: Perfil estratigráfico Oeste de la unidad de excavación 3.

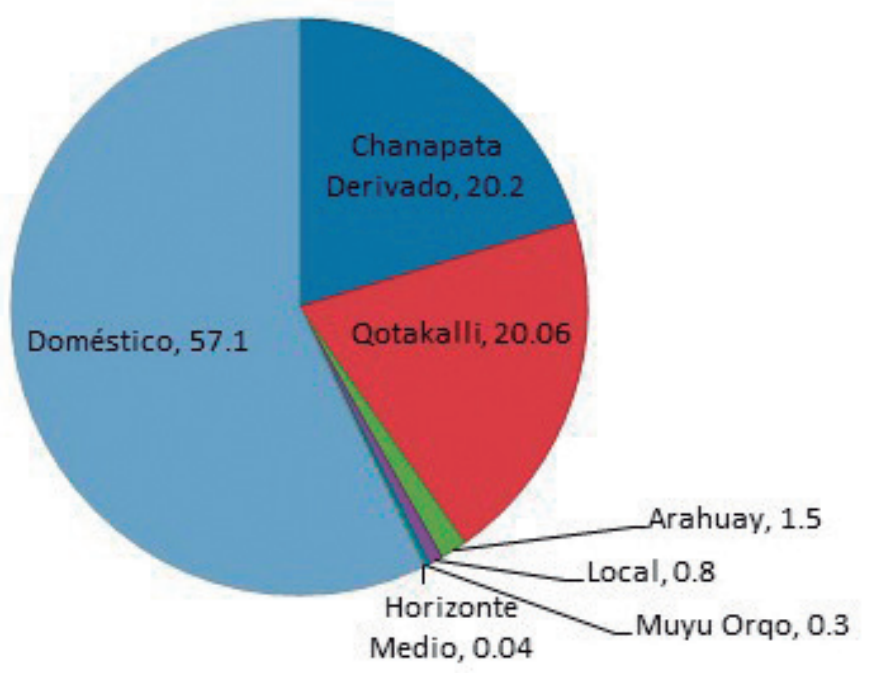

Figura 5: Porcentajes de estilos cerámicos de la Unidad de excavación 3.

7. El total de fragmentos cerámicos analizados en la Unidad de Excavación $\mathrm{N}^{\circ} 3$ fue de 2,867. 


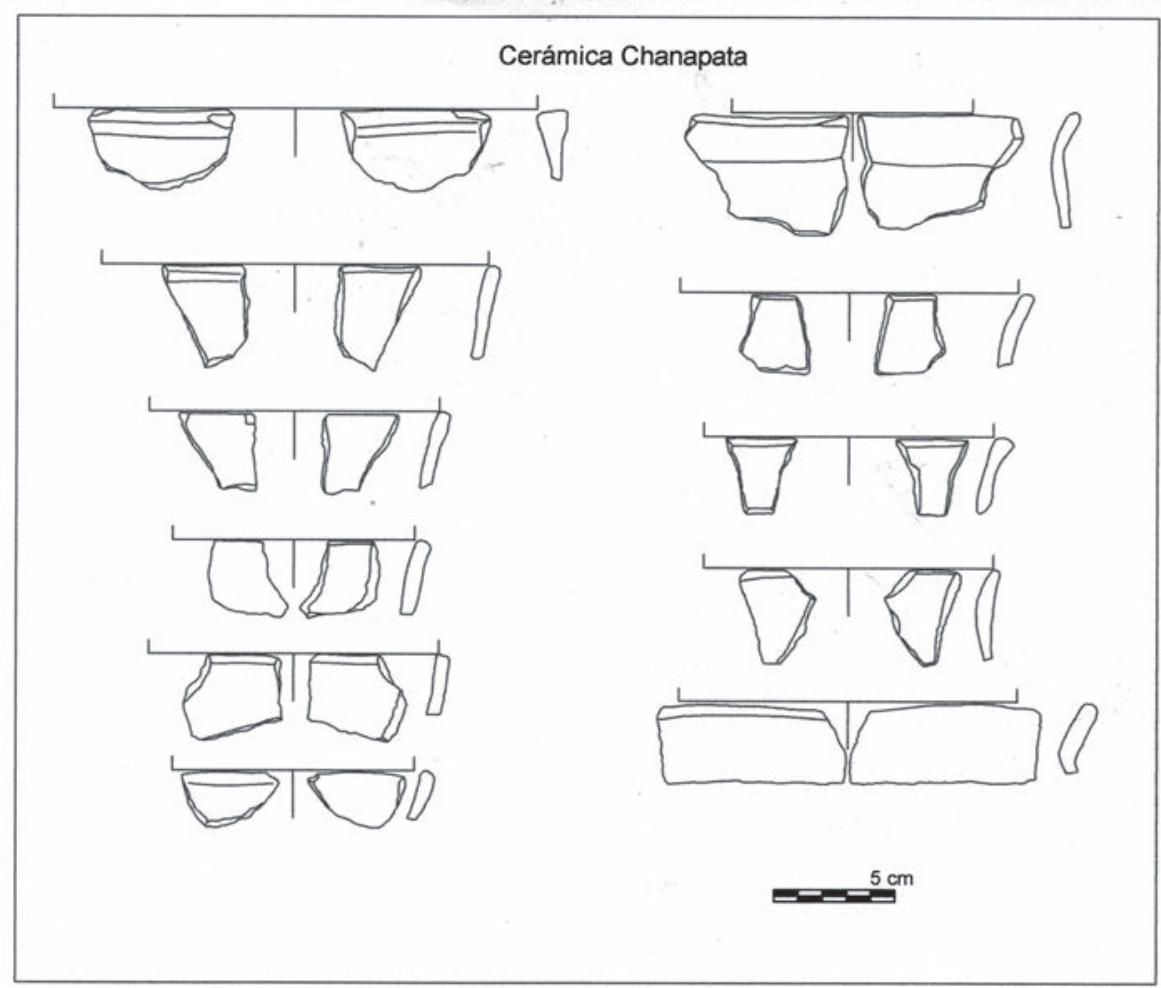

Figura 6. Cerámica Chanapata.

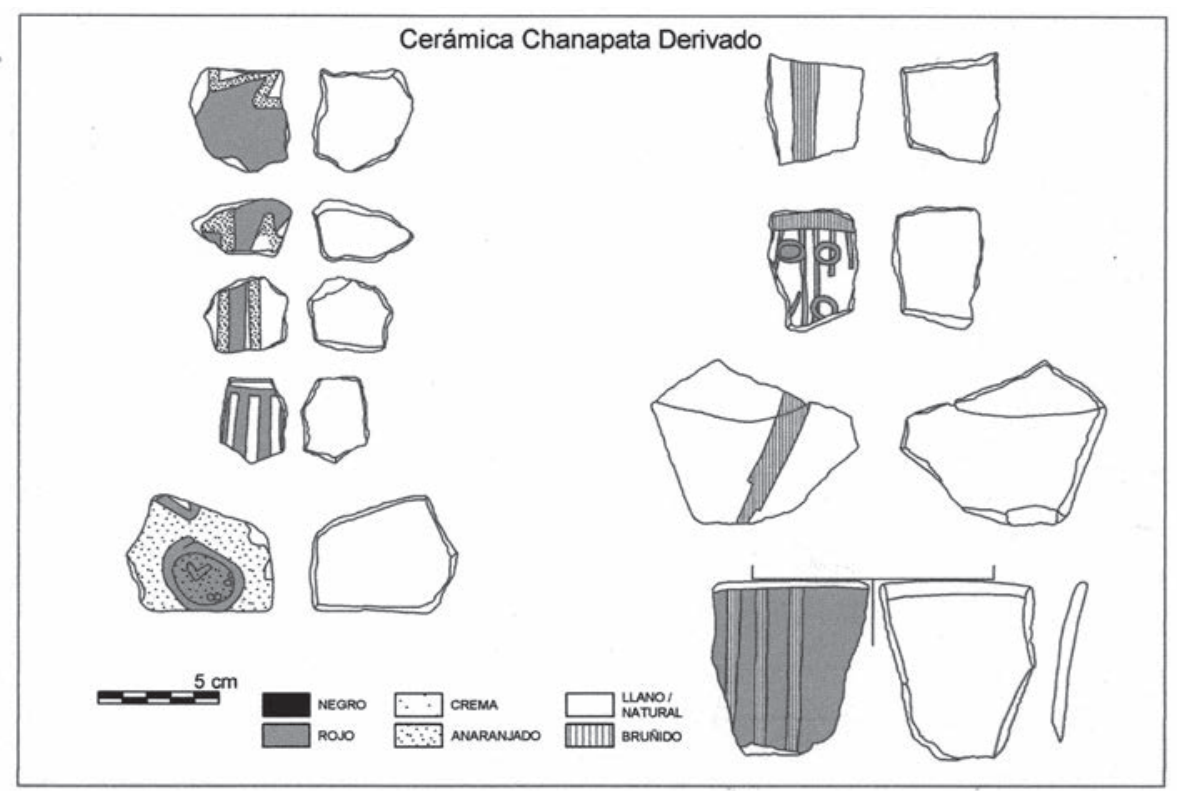

Figura 7. Ceramica Chanapata y Cerámica "Local". 

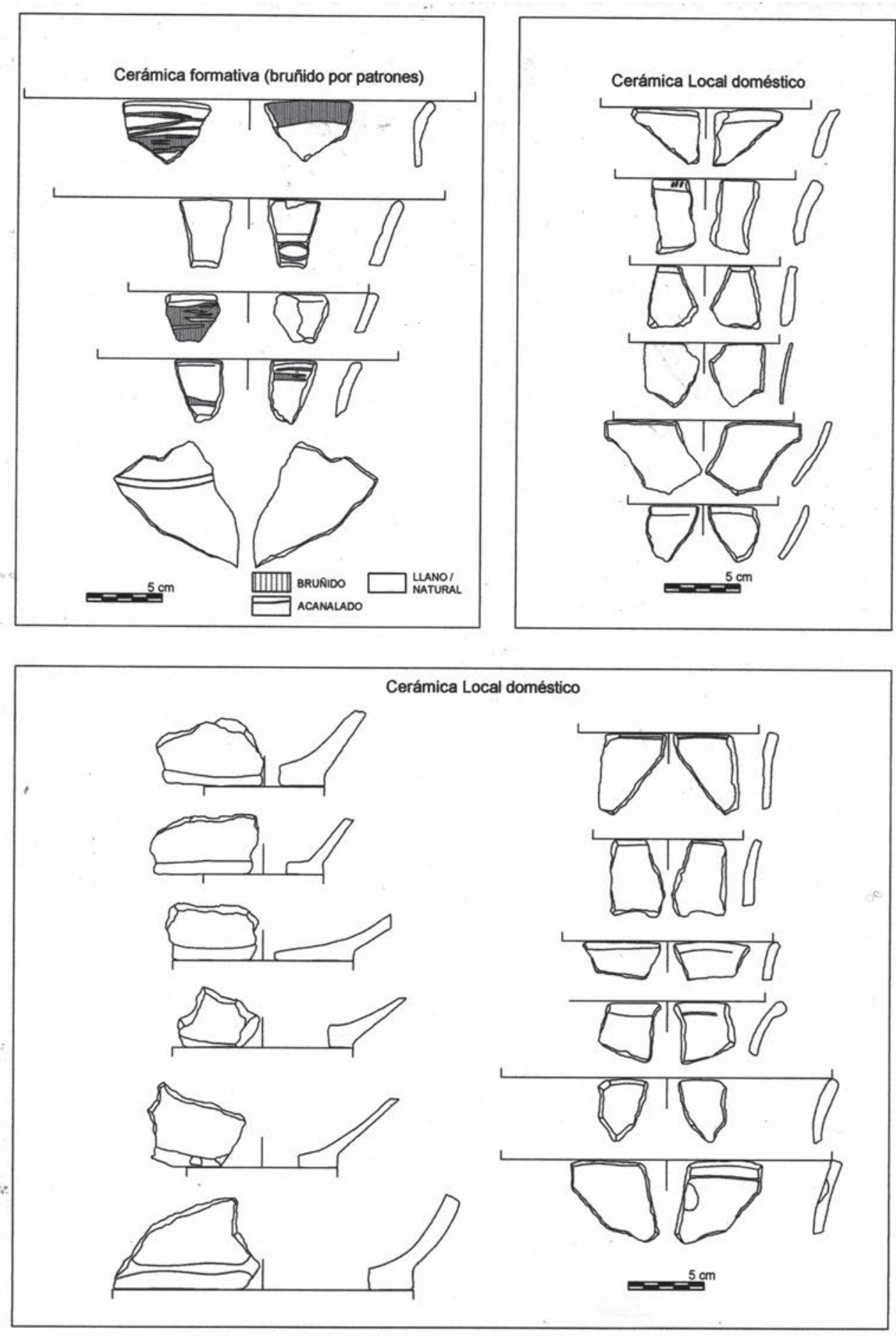

Figura 8. Cerámica decorada "Local". 


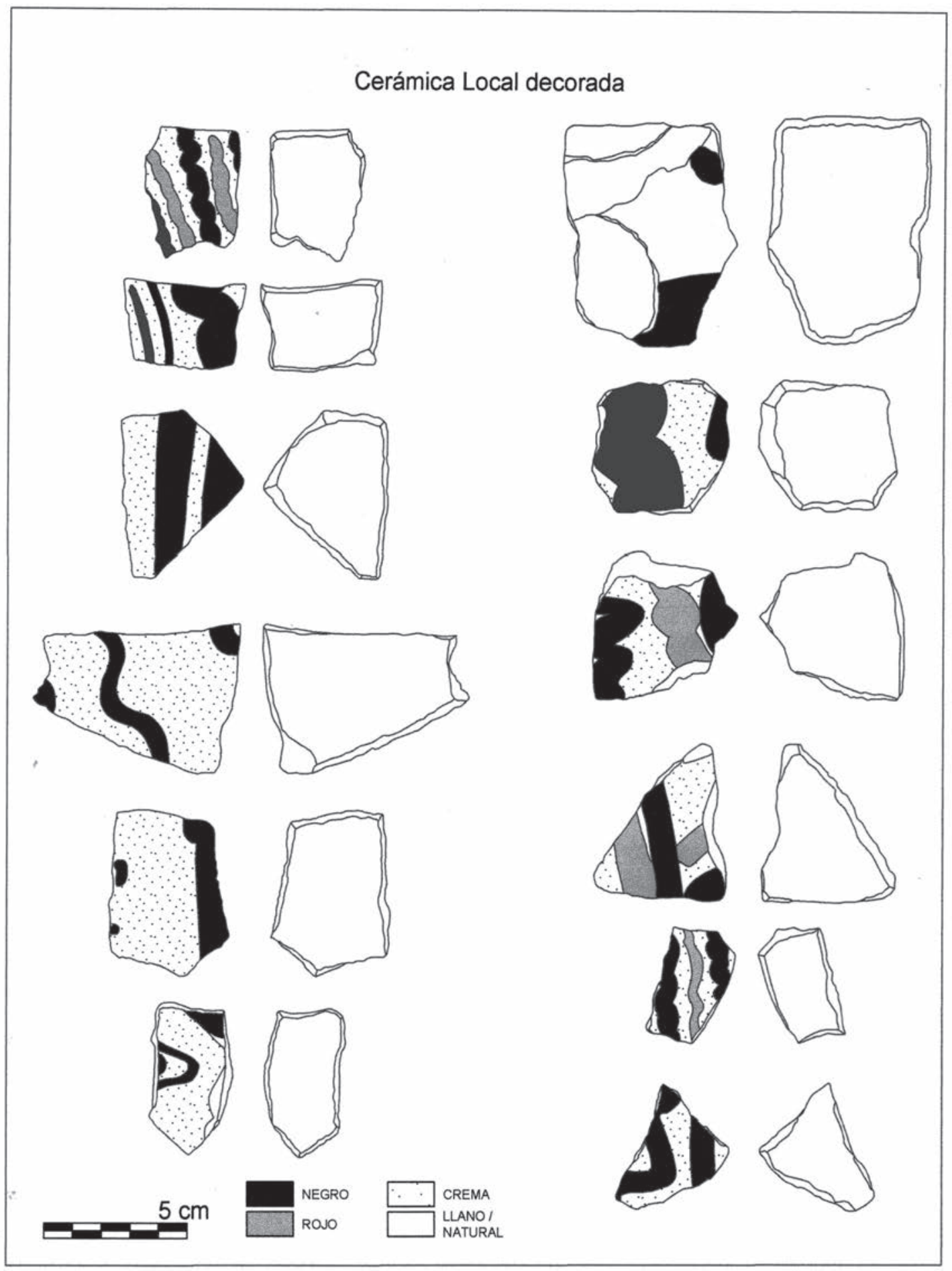

Figura 9. Cerámica Qotakalli. 


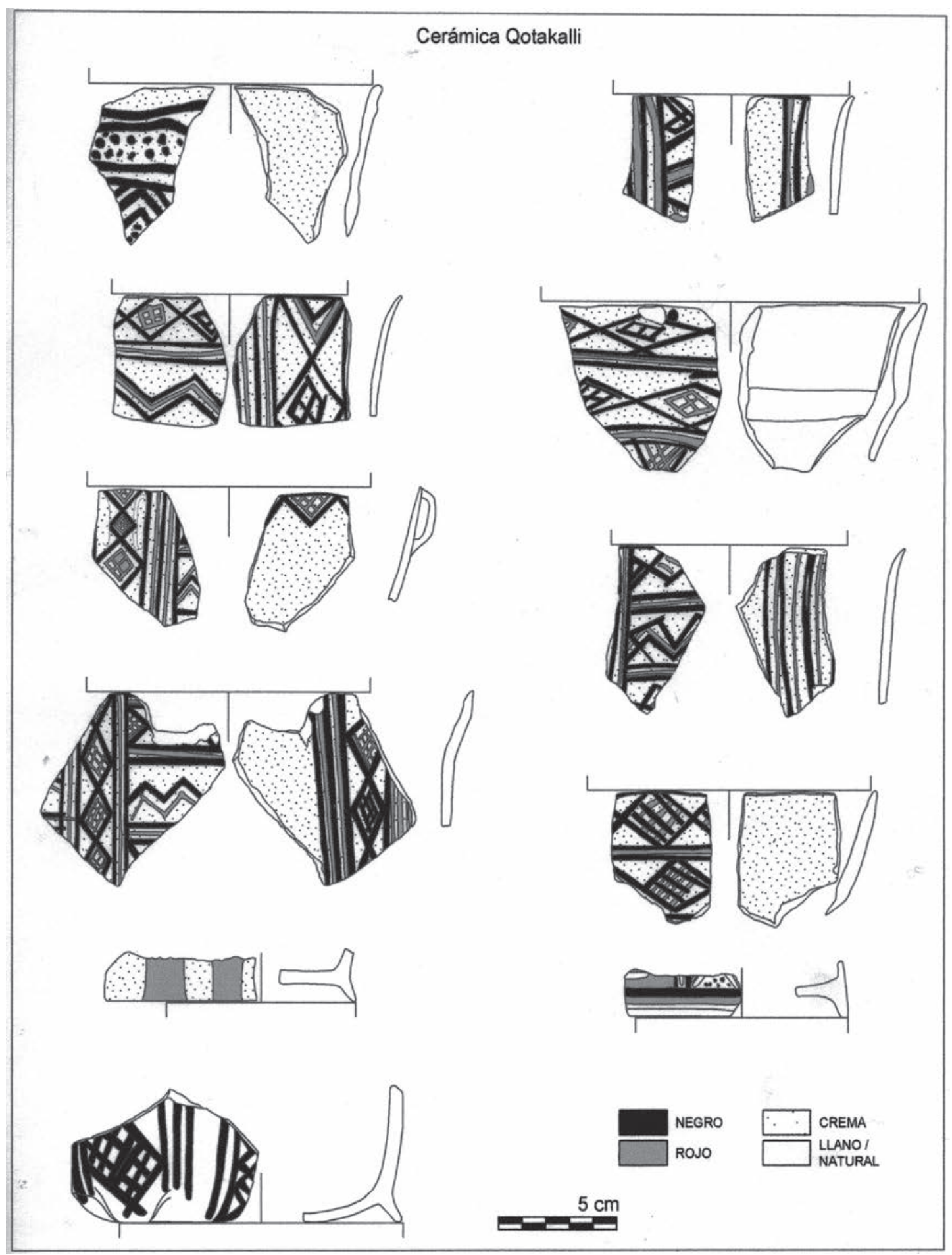


Asociada al estilo de cerámica Qotakalli se obtuvo una muestra de madera que nos dio un fechado radio carbónico que corresponde al período Intermedio Temprano/Horizonte Medio. Asociado a esta muestra se tiene un estilo de cerámica que denominamos "local" que corresponde al Intermedio Temprano/Horizonte Medio (ver fechado radiocarbónico).

Muestra Años C14

AA101203 Madera
$1426+-36$
$95 \%$
Años calendarios calibrada $592-762$ cal DC $^{8}$

Este fechado radiocarbónico es consistente con los obtenidos en Peqokaypata y Tankarpata, ambos sitios asociados con cerámica Qotakalli (Bauer 2008:374) y en el sitio de Ak'awillay Pampa de Anta (Bélisle 2015).

Luis Barreda fue quien realiza la primera clasificación del estilo Qotakalli en 7 tipos (1982:18), posteriormente Bauer ubica 115 sitios asociados a cerámica Qotakalli en el valle del Huatanay (2008: 109). Su centro de producción se ubicaría en el valle del Huatanay (Bauer y Jones 2011, Covey et al. 2013), abarcaría algunas áreas vecinas como la Pampa de Anta (Belisle y Galiano 2010, Belisle y Covey 2010 y Belisle 2011), la provincia de Paruro (Bauer 2008) y el Valle Sagrado (Kendall 1976b, Covey et. al 2008).

El estilo Qotakalli tiene una duración bastante larga desde el período Intermedio Temprano (AD 200-600) y su producción continuó durante el Horizonte Medio. (Belisle y Covey 2010:82). Es probable que en Conventomoqo esto este sucediendo debido a que tenemos algunos fragmentos Muyu Orqo.

Bauer fue el primero que identifica el estilo Muyu Orqo y sugiere una posible influencia Tiahuanaco y lo ubica en el Horizonte Medio (Bauer 2002, 2008). Espinoza (1983) encuentra fragmentos de cerámica "Wari de Aqomoqo" en sus excavaciones en el sitio de Aqomoqo que se encuentra a la margen derecha del riachuelo de Cebollahuayco. Para Bauer estos fragmentos encontrados por Espinoza corresponderían a Muyu Urqo (2002:106).

En la pampa de Xaquijahuana el sitio de Ak'awillay tiene una ocupación desde el período Formativo y continua en el Intermedio Temprano y Horizonte Medio con un área de 10 hectáreas, lo que es el sitio más grande de esta área (Bélisle 2011, Belisle y Covey 2010:84). A base de fechados radiocarbónicos, los estilos cerámicos del período Intermedio Temprano empezaron a ser producidos aproximadamente 200-600 d.C, pero su uso también siguió durante la primera mitad del Horizonte Medio (aproximadamente 600-800 d.C) y tal vez más tarde (Bélisle 2011; Bélisle y Galiano 2010: 149).

La unidad 3 de Conventomoqo corresponde a un basural, donde la cerámica Chanapata se encuentra mezclada con cerámica Qotakalli, y un tipo al que denominamos "local" que probablemente corresponda al Período Intermedio Temprano. Esta misma situación se dio en algunas casas excavadas en Ak'awillay (Belisle y Covey 2010:84 88). Los estilos cerámicos pueden seguir siendo producidos y utilizados por mucho más tiempo, entonces es probable que la cerámica Chanapata se siguió produciendo durante el periodo de uso del estilo Qotakalli con muy pocas diferencias, como se puede ver en Ak'awillay (Bélisle y Galiano 2010:147). En la costa norte del Perú se tiene una situación similar donde la cerámica Gallinazo sigue siendo usada mientras la cerámica Mochica es producida (Millaire y Morlion 2009).

Bauer y Jones excavaron los sitios arqueológicos de Tankarpata, Peqokaypata y Pukakancha en el valle del Cusco encontrando varios contextos de cerámica Arahuay mezclada con cerámica Qotakalli. Lo interesante fue que en todos se tomaron muestras radiocarbónicas y el fechado de Pukakancha entre 430-720 D.C parece que podría estar marcando el inicio del uso de cerámica Qotakalli con Arahuay

8. Laboratory number: AA101204 NSF Arizona AMS Laboratory 
(Bauer y Jones 2011:143), este uso de ambos estilos parece que perduró por varios siglos más durante el período Horizonte Medio.

\section{AnÁlisis CERÁmico}

El estudio arqueométrico de la cerámica lo realizó el Ing. Jorge Gamarra que consiste en el examen mineralógico, petrográfico y químico de la materia prima. Se analizaron 12 muestras de los estilos cerámicos Chanapata, Qotakalli, y Local (Gamarra 2013). Todas las muestras mostraron una misma matriz arcillosa procedentes de una arcilla limosa cuya matriz litológica procede de una roca arenisca de grano medio y fino, clastos sedimentarios de caliza de la formación geológica San Sebastián y San Jerónimo. La diferenciación de los antiplásticos si es variable entre las muestras analizadas.

Las muestras cerámicas Chanapata tienen pequeñas diferencias entre ellas de su matriz arcillosa: arenisca, feldespática y arenisca calcita, asimismo, el material antiplástico son del tipo cuarcitica.

Las muestras Qotakalli tienen una misma matriz arcillosa de arenisca cuarcitica, pero si tienen diferencias en la adición del material no plástico (cuarcita, andesita roja y granito) lo que supondría que estos materiales proceden de canteras distintas, esto no quiere decir que tengas diferencias cronológicas.

La cerámica denominada "Local" es de pasta oxidada de color anaranjada o marrón, de acabado tosco. La decoración consiste en líneas gruesas onduladas pintadas descuidadamente de color negro, rojo, naranja sobre un fondo natural (ver figuras $\mathrm{N}^{\circ} 7$ y 8 ). El tratamiento de la superficie externa es alisado y la interna es trapeada. La pasta tiene una matriz arcillosa de roca arcillosa y la matriz no plástica de roca arenisca cuarcitica.

Todo este análisis nos ha permitido determinar que la cerámica de Conventomoqo de estos estilos son de fabricación local, se ha utilizado las canteras de arcilla del mismo lugar. Esto nos habre la posibilidad que el lugar no fue ocupado por un nuevo grupo proveniente de otro lugar y que como parte de su nuevo asentamiento introdujeron nuevos elementos. Es lógico pensar que las poblaciones que estuvieron asentadas en el lugar durante el último momento del período Horizonte Temprano continuaron durante el Intermedio Temprano y solamente incorporaron nuevos elementos cerámicos como el estilo Qotakalli, Arahuay y el estilo de franjas gruesas y onduladas que estamos denominando Local.

La cerámica Chanapata Derivado se encuentra uniformente distribuido en toda la colina del cerro Contaymoqo, el estilo Qotakalli no aparece de manera uniforme en todo el área, sino más bien se encuentra focalizado en algunos sectores. Esto no quiere decir que la incorporación de nuevos elementos implique necesariamente cambios en su organización social, económica, estos cambios no se han podido definir en las excavaciones arqueológicas.

\section{LA DiETA}

De acuerdo a los análisis realizados en Conventomoqo la dieta estuvo basada en quínua, Kiwicha, maíz (Rojas 2011), para este período ya se han identificado el consumo de quinua (Davis y Delgado 2010: 352, Chepstow-Lusty 2011: 571, Bauer 2008: 57), el maíz igualmente en Yuthu era parte de la dieta y para Chepstow-Lusty el período Formativo Tardío sería el inicio de la agricultura importante (2011: 576). Asimismo, otra muestra analizada en el Museo de Historia Natural UNMSM - LIAP identificó semillas de la familia solanácea que podrían corresponder a semillas de papa o ají silvestre 
(Bertoni 2012). El consumo de papa y otros tubérculos todavía es discutible y se necesitan mayores datos para esta afirmación. Complementando la dieta el consumo de camélidos y roedores como el Cuy (cavia porcellus) es consistente.

\section{Comentarios Finales}

En el valle del Huatanay el numero de los sitios arqueologicos del período Formativo estarían por encima de los ochenta y mayormente corresponden al Formativo Tardío (Bauer 2008:88). Estos se encuentran generalmente en la margen derecha del río Huatanay y Conventomoqo se enconraría a 6 $\mathrm{km}$ de distancia de los sitios más grandes de Wimpillay y Muyu Orqo. El lugar se emplaza sobre una loma parcialmente modificada, esta es una característica que se da durante el Formativo y en otros períodos.

Posterior a esta ocupación formativa en algunos espacios en Conventomoqo tenemos una ocupación del período Intermedio Temprano final con el Horizonte Medio asociada a cerámica Qotakalli y una cerámica que tentativamente la denominamos local, por sus semejanzas con la cerámica Local que determinó Belisle y Galiano (2010) en la Pampa de Anta. Lo interesante es notar que estos estilos cerámicos se encuentran mezclados con Chanapata Derivado, lo que nos indicaría que ambos estilos estuvieron produciendose conjuntamente. Las tradiciones alfareras locales tuvieron una continuidad en el tiempo a pesar de la presencia Wari en Lucre y Huaro al sur del Valle del Cusco.

Asimismo, es importante saber que los alfareros de Conventomoqo desde el período Formativo hasta el Horizonte Medio estuvieron produciendo su cerámica con la arcilla de la zona y no se tiene cerámica importada de otras regiones.

\section{BibLIOGRAFÍA}

BARREDA, Luis

1973 Las culturas Inka y Pre Inka del Cusco. Tesis para optar el grado de Doctor en Letras y Ciencias Humanas. Universidad Nacional San Antonio Abad del Cuzco.

1982 “Asentamiento humano de los Qotakalli del Cuzco". Arqueología del Cuzco, 13-20. Instituto Nacional de Cultura Región Cusco.

BAUER, Brian

2002 Las antiguas tradiciones alfareras de la región del Cuzco. Cuzco: Centro Bartolomé de las Casas.

2008 Cuzco Antiguo: Tierra natal de los Incas. Cusco: Centro Bartolomé de las Casas.

BAUER, Brian y Bradford JONES

2011 "Estilos cerámicos del período Intermedio Temprano y el Horizonte Medio en el valle del Cuzco”. Estudios Arqueológicos sobre los Incas, 127-170. Cusco: Centro de Estudios Regionales Andinos Bartolomé de las Casas.

BÉLISLE, Véronique

2011 Ak'awillay: Wari state expansion and household change in Cusco, Peru (AD 600-1000). Dissertation submitted in partial fulfillment of the requirements for the degree of Doctor of Philosophy (Anthropology). University of Michigan.

2015 "Understanding Wari State Expansion: A “Bottom-Up”. Approach at the Village of Ak'awillay, Cusco, Peru”. En: Latin American Antiquity, 26(2): 180-199. SAA. 
BÉLISLE, Véronique y Vicentina GALIANO

2010 “Ak'awillay: un pueblo autónomo durante la colonización Wari en el Cusco”. Revista Andina, 50: 141-173. Cusco: Centro de Estudios Regionales Andinos Bartolomé de las Casas.

BÉLISLE, Véronique y Alan COVEY

2010 "Local settlement continuity and Wari impact in Middle Horizon Cusco". Beyond Wari wall; regional perpectives on middle Horizon Perú. 79-95. Albuquerque: Universidad of New Mexico.

BERTONI, Gabriela

2012ms Informe Arqueo botánico Altiva Canas, Cusco. Museo de Historia Natural UNMSM - LIAP Laboratorio de Investigaciones Arqueo botánicas del Perú.

\section{CENTRO GUAMAN POMA DE AYALA}

2005 Amanecer en el Bajo Huatanay. C.E.C Guaman Poma de Ayala.

CHÁVEZ, Karen

1980 "The archaeology of Marcavalle, an early horizon site in the valley of Cuzco, Peru: Part I". Baessler-Archiv N.F. 28: 203-329.

1981a “The archaeology of Marcavalle, an early horizon site in the valley of Cuzco, Peru. Part II". Baessler-Archiv N.F. 29: 107-205.

$1981 b$ “The archaeology of Marcavalle, an early horizon site in the valley of Cuzco, Peru. Part III". Baessler-Archiv N.F. 29: 241-386.

1982 "Resumen de los trabajos arqueológicos realizados en Marcavalle, un sitio correspondiente al Horizonte Temprano en el valle del Cuzco". Arqueología del Cuzco. 1-8. Cusco: Instituto Nacional de Cultura.

CHEPSTOW-LUSTY, A.

2011 "Agro-pastoralism and social change in the Cuzco heartland of Peru: a brief history using environmental proxies”. Antiquity, 85, 570-582.

COVEY, Alan

2003 "The Vilcanota Valley (Perú): Inka state formation and the evolution of imperial strategies". Tesis de Doctorado. University of Michigan, Departament of Antropology.

COVEY, Alan; ARAÓZ M; BAUER, B.

2008 Settlement Patterns in the Yucay and neighboring areas. Imperial Transformations in sixteenth-century Yucay, Perú. 3-17. Studies in Latin American Ethohistory \& Archaeology. Ann Arbor Michigan.

DAVIS, Allison y Carlos DELGADO

2010 "Investigaciones arqueológicas en Yuthu: nuevos datos sobre el período formativo en el Cusco, Perú (400-100 a.C)”. Boletín de Arqueología,13: 347-372. Pontifica Universidad Católica del Perú.

DELGADO, Carlos

2014ms “Informe final del Proyecto de Investigaciones Arqueológicas en Bandojan”, primera temporada. 
DWYER, Edward

1971 “A Chanapata figure from Cuzco, Perú”. Nawpa Pacha, 9: 33-40, Berkeley.

ESPINOZA, Héctor

1983 “Evidencia cultural el Horizonte Medio (Wari), Aqomoqo-Cusco". Arqueología Andina, 16-22. Cusco: Talleres Gráficos del instituto Nacional de Cultura Departamental Cusco.

GAMARRA, Jorge

2013ms Resultados de análisis de cerámica Conventomoqo. Cusco.

GLOWACKI, Mary

1996 "The Wari occupation of the southern highlandsof Perú: A ceramic perspective from the side of Pikillacta". Tesis de Doctorado. The Faculty of the Graduate School of Arts and Sciences Brandeis University, Department of Anthropology.

KENDALL, Ann

1976 "Preliminary report on ceramic data and the Pre-Inca architectural remains of the (lower) Urubamba valley, Cuzco”. Baessler-Archiv, Band, XXIV, 41-159 Neue Folge.

MCEWAN, Gordon

1984 “Investigaciones en la Cuenca del Lucre, Cusco". Gaceta Arqueológica Andina, 9, 12-15. Lima

MILLAIRE, Jean-Francois y Magali MORLION

2009 Gallinazo: An early cultural tradition on the Peruvian North Coast. Los Angeles: Cotsen Institute of Archaeology Press. University of California.

ROJAS, Eliana

2011ms "Informe con resultados de análisis de rastreo e identificación de materia orgánica en muestras del PEA Altiva Canas - Cusco”. Informe № 003-2011-ERV-DFQ-SDI-DIC-DRC-C/MC

ROWE, John H.

1944 "An introduction to the archaeology of Cuzco". Papers of the Peabody Museum of American Archaeology and Ethnology, 10-23. Harvard University, Cambridge, MA.

TORRES, Nilo

1989 "Sondeo arqueológico de Araway". Tesis para optar al grado de Licenciado en Arqueología, Facultad de Ciencias Sociales, Universidad Nacional de San Antonio Abad del Cusco.

UGENT Donald y Carlos OCHOA

2006 La Etnobotánica del Perú, desde la prehistoria al presente. Lima: Concytec.

YÁBAR, Jorge

1959 “La cultura pre-Incaica de Chanapata”. Revista del Museo e Instituto Arqueológico, 18: 93-100.

1972 “Época pre-Inca de Chanapata”. Revista Saqsaywaman, 2: 211-233, Patronato Departamental de Arqueología del Cusco. 
1982 “Figurillas de la cultura Pre-Inka del Cuzco". Arqueología de Cuzco, 9-12. Instituto Nacional de Cultura, Cusco.

ZAPATA, Julinho

1997 “Arquitectura y contextos funerarios Wari en Batan Urqu, Cusco”. Boletin de Arqueología, 1: 165-206. Pontifica Universidad Católica del Perú.

1998 "Los cerros sagrados: Panorama del periodo formativo en la cuenca del Vilcanota, Cuzco". Boletin de Arqueología. № 2: 307-336. Pontifica Universidad Católica del Perú. 